Article

\title{
A Pilot Randomised Controlled Trial to Enhance Well-Being and Performance of Athletes in Para Sports
}

\author{
Hannah Macdougall ${ }^{*}$, Paul O'Halloran ${ }^{2}$, Emma Sherry $^{3}$ and Nora Shields ${ }^{4}$
}

Received: 23 ${ }^{\text {rd }}$ September 2018; Accepted: 5 ${ }^{\text {th }}$ April 2019; Published: 7th August 2019

\begin{abstract}
Existing mindfulness and acceptance-commitment programs in elite sport have ignored elite athletes with a physical impairment. A pilot randomised controlled trial was conducted to determine if an eight-session mindfulness-acceptance-commitment program delivered within a motivational interviewing framework would enhance well-being for athletes in Para Sports. Eighteen athletes in Para Sports were randomly assigned to either intervention $(n=9)$ or waitlist control ( $n=9$ ) groups, with the intervention group attending eight 60 -minute face-to-face individual sessions. Significant improvements were identified in the intervention group for the primary outcomes of satisfaction with life (subjective well-being), personal growth, positive relation with others (psychological well-being) with large effect sizes (i.e., partial eta squared range $=.23$ to .30 ) when compared to the waitlist control. Significant improvements were also identified in the intervention group for a reduction in perceptions of pain (physical well-being), a secondary outcome, when compared to the waitlist control group. Although not statistically significant, moderate to large effect sizes in favour of the intervention group were also calculated through this trial for multiple domains of subjective and psychological well-being. No significant or practically meaningful effect sizes were calculated for social well-being. Through the results of this study, we have presented preliminary evidence for combining mindfulness-acceptance interventions with motivational interviewing, which we demonstrate can result in meaningful well-being improvements. Future research is needed to confirm the efficacy of this approach in adequately powered sample sizes.
\end{abstract}

Keywords: acceptance and commitment therapy, elite athletes, disability, intervention, mindfulness, motivational interviewing, Paralympics, physical impairment.

\section{Introduction}

Elite athletes are required to maintain exceptional standards of performance with high training loads and short periods of recovery time (Ho, Appleton, Cumming, \& Duda, 2015). Within this context, they are also subjected to environments and stressors that can hinder well-being and lead to burnout, high levels of negative affect, and debilitating mood changes (Lemyre, Hall, \& Roberts, 2008). The authors adopted a person-first language approach and used the term 'athletes in Para Sports' to refer to athletes who compete in Paralympic sport but may not necessarily have competed at the Paralympic games. For athletes in Para Sport competing in the Paralympics, due to various additional physical considerations, having strong psychological skills and well-being is particularly important (Macdougall, O'Halloran, Sherry, \& Shields, 2016; Martin, 2012). For example, athletes in Para Sports face challenging situations to travel to events inclusive of transfers from wheelchairs to plane seats and bathroom accessibility (Campbell \& Jones, 2002). Athletes in Para Sports may experience stress during classification processes and setbacks associated with this or during a drug testing experience complete with the added complications of a catheter (Martin, 2012; Powell \& Myers, 2017). These examples can be further amplified through a complex interaction of physical pain, trauma and recovery, difficulties with emotional regulation, and a lack of self-acceptance 
(Macdougall et al., 2016; Powell \& Myers, 2017). However, documented strengths of athletes in Para Sports include personal growth, optimism, resilience, strong social support networks, meaningful contributions to multiple communities, and mental toughness (Macdougall et al., 2016; Powell \& Myers, 2017). These global well-being strengths have been found to be foundational for sport-specific well-being (Macdougall et al., 2016); foundational qualities may not directly influence performance, but they often have an indirect impact through training quality, social support, and lifestyle choices (Lundqvist \& Sandin, 2014; Martin, 2012). Thus, interventions that look to enhance the well-being of athletes in Para Sports may also favourably impact performance (Macdougall et al., 2016).

A prominent approach to investigate well-being, and the approach used to define well-being in the current study, is integrating the two theoretical traditions of hedonia and eudaimonia (Macdougall et al., 2016; Lundqvist \& Sandin, 2014; Ryan \& Deci, 2001). As seen in Figure 1, the hedonic tradition is associated with subjective well-being, and the dimensions of long-term affect and satisfaction with life (Diener, Emmons, Larsen, \& Griffen, 1985). The eudaimonic tradition is connected with psychological and social well-being (Lundqvist \& Sandin, 2014), whereby psychological well-being incorporates autonomy, environmental mastery, personal growth, positive relation with others, purpose in life, and self-acceptance (Ryff, 1989), and social well-being incorporates acceptance, actualisation, coherence, contribution, and integration (Keyes, 1998).

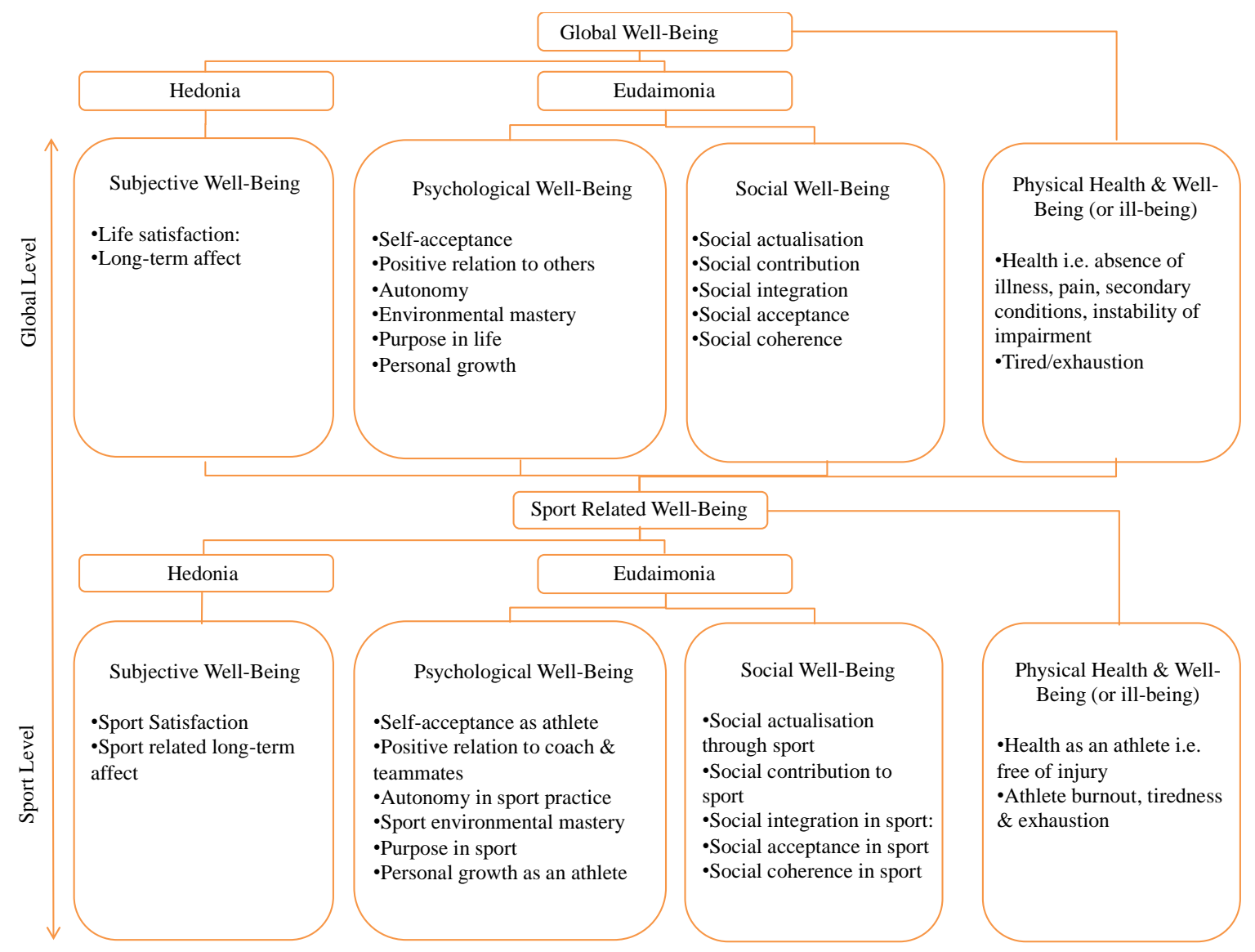

Figure 1: An integrated model of athletes in Para Sports health and well-being in a global and sportrelated context (adapted from Macdougall et al., 2016; Lundqvist, 2011)

The well-being framework, depicted in Figure 1, was initially developed within the theoretical review by Lundqvist (2011). Lundqvist (2011) identified the ambiguous and inconsistent definitions of well-being used within sport psychology and addressed this through contextualising well-being at a global and sport-specific context using the theoretical traditions of hedonia and eudaimonia. The framework has since been used to qualitatively investigate the well-being of athletes in Para sports by Macdougall and colleagues (2016). Within this research, the relevance of physical health and well- 
being was recognised for athletes in Para Sports, and thus saw the framework modified to include this aspect. While Macdougall and colleagues (2016) found that contextualising well-being provided helpful insights into the well-being of athletes in Para Sports, this paper has been purposefully delimited to global well-being.

Within sport, third-wave cognitive approaches such as mindfulness and acceptance and commitment therapy (ACT) interventions have been shown to improve performance and aspects of well-being (e.g., mindfulness; Bernier et al., 2009; Gardner \& Moore, 2004). The Mindfulness Acceptance Commitment (MAC) for performance enhancement program was developed specifically for athletes to provide an alternative to traditional skills-training approaches (Gardner \& Moore, 2004). The MAC program is an integration of ACT (Hayes, Strosahl, \& Wilson, 1999) and mindfulness-based stress reduction (Segal, Williams, \& Teasedale, 2002) for use with athlete populations. According to these two approaches, optimal performance does not call for a reduction or volitional control of internal states. Rather, it requires (a) non-judgment, moment-to-moment awareness and acceptance of internal states, (b) attentional focus on task-relevant external stimuli, and (c) consistent and effortful personal values-driven commitment to behavioural actions that support athletic endeavors (Gardner \& Moore, 2012).

Since it was developed, the MAC program has gained preliminary empirical support for performance and mindfulness enhancement through randomised controlled trials (RCTs) and casestudies within sport (see Gardner \& Moore, 2012, for review). For example, Goodman et al. (2014) found a MAC and yoga-based intervention for a National Collegiate Athletics Association Division I athletic team resulted in greater mindfulness compared to a non-randomised controlled group. Other studies where the MAC program have been applied have reported improvements in the areas of mindfulness and flow (e.g., Thompson, Kaufman, De Petrillo, Glass, \& Arnkoff, 2011), yet have not applied the theoretical frameworks of hedonia or eudaimonia, and their respective measures, to examine changes in well-being. This is surprising given mindfulness and ACT interventions have been shown to increase subjective well-being (Grossman, Niemann, Schmidt, \& Walach, 2004), psychological well-being (Brown \& Ryan, 2003), and physical well-being (Veehof, Oskam, Schreurs, \& Bohlmeijer, 2011), in clinical and non-clinical populations.

As well, the extant literature is yet to include elite (i.e., national level and above) athletes in Para Sports. It is important to emphasise that research focusing on athletes in Para Sports does not seek to create the impression that athletes in Para Sports are not elite or are separate from elite, able-bodied athletes. Especially given that elite athletes in Para and Olympics sports share many similarities (e.g., dimensions of long-term affect; see Macdougall, O'Halloran, Shields, \& Sherry, 2015, for review). However, as highlighted previously, due to various additional physical considerations, and challenges, athletes in Para Sports face unique experiences. For example, the well-being strengths (i.e., areas of high well-being) of athletes in Para sports include accepting the nature of their impairments and psychological flexibility (Macdougall et al., 2016; Martin, 2012). As such, the unique challenges and well-being strengths of athletes in Para Sports indicate an opportunity for a MAC intervention to focus upon athletes in Para Sports.

The MAC program has been previously combined with other therapies (e.g., Yoga, Goodman et al., 2014), and program components, such as ACT, and could potentially be enhanced by motivational interviewing (MI; Bricker \& Tollinson, 2011). MI is a person-centered approach used to address ambivalence about change through facilitating and engaging intrinsic motivation (Miller \& Rollnick, 2012). MI shares many similarities with the MAC program. For example, an attitude of collaboration, autonomy within the partnership, and connecting with values (Bricker \& Tollison, 2011; Miller \& Rollnick, 2012). In contrast, differences between MAC and MI include emphasis on language process vs. language content, and application of metaphors/experiential exercises vs. open-ended questions, affirmations, and reflections (see Method for details; Bricker \& Tollison, 2011; Miller \& Rollnick, 2012). Thus, MI is compatible to MAC given the similarities in the spirit in which it is delivered. However, the emphasis on core micro skills such as the use of reflections in MI suggests it could offer some additional benefits to MAC alone. 
MI has a strong base of evidence for its effectiveness. Clinical trials have found MI promoted treatment engagement, retention, and adherence with large effect sizes (Hettema, Steele, \& Miller, 2005). Similar effect sizes have been reported when MI is added to other active psychotherapieseach enhancing the impact of the other (Csillik, 2015). As such, it is possible that MI would maintain the athletes' engagement with the MAC program and adoption of behaviour patterns learned during MAC.

The purpose of this trial was to explore the feasibility and potential efficacy of MAC delivered within an MI framework. The trial aimed at improving perceived well-being dimensions relevant for athletes in Para Sports with physical impairments (Macdougall et al., 2016). Applying these specific well-being dimensions would help to address the lack of comprehensive application of hedonic or eudaimonic traditions when MAC has been delivered. The primary hypothesis (H1) for the study was that participants in the intervention group would have larger improvements in subjective (hedonia; H1a), psychological (H1b), and social well-being (eudaimonia; H1c) than participants in the wait list control group. The secondary hypothesis (H2), related to the MAC program, was that participants in the intervention group would have larger improvements in perceived mindfulness (H2a) and acceptance (H2b) than participants in the wait list control group. Distinct from hedonia and eudaimonia, but relevant for athletes in Para Sports, it was also hypothesised (H3) that participants in the intervention group would have larger improvements in perceived physical wellbeing or health (i.e., perceived pain and burnout; Macdougall et al., 2016) compared to the wait list control group. In sum, these hypotheses are consistent with improving the well-being strengths of athletes in Para Sports previously identified (Macdougall et al., 2016), rather than address a specific challenge per se.

\section{Materials and Methods}

\section{Trial Design}

A repeated-measures RCT with concealed allocation was conducted. The trial was prospectively registered with the Australian and New Zealand Clinical Trials registry (ANZCTR No. 369377). Participants allocated to the intervention group received the MAC program delivered within an MI framework and participants allocated to the wait list control group continued their usual activities for eight weeks. Ethical approval was obtained from the University Ethics Committee and participants gave their written informed consent to participate. The consent form included a protocol for adverse events and support resources.

\section{Participants}

Participants were included if their age was over 18 years, competing at national level or above in a Paralympic sport (please see the classification system of the International Paralympic Committee for details regarding the classification system and eligible impairments; IPC, 2016), and had a physical impairment with no secondary impairment (e.g., sensory or intellectual impairment). Participants were also required to speak English, and be able to attend eight weekly individual sessions located in Victoria, Australia. Participants were recruited via email through the Victorian State Institute of Sport (VIS). VIS staff sent an email to athletes in Para Sports currently on scholarship. The email sent to athletes included the promotional material for a well-being and performance enhancement trial and those interested in participating were encouraged to contact the researchers directly. As participants contacted the researcher directly, this ensured the confidentiality and anonymity of participates from Institute staff members. This approach was complimented through a social media campaign using Facebook, Twitter, and Instagram, which saw similar promotion details (e.g., study detail, duration, location, etc.) included across social media platforms. Participants were screened before enrolment via phone, skype, or face-to-face, to ensure they met the inclusion criteria. During screening sessions, the researcher emphasised the voluntary nature of the study and assured participants of their confidentiality and anonymity. Participants were informed of their ability to withdraw from the study at any time up until four weeks after the conclusion of the intervention. A 
member of the research team not involved in recruitment, assessment, or intervention, randomly assigned participants after baseline assessment through a concealed allocation block randomised method, (blocks of four), to either the intervention group or wait list control group using a random number generator. Participants did not receive any form of compensation.

\section{Intervention}

The procedures outlined by Gardner and Moore (2007; see Table 1) were followed to deliver the eight-module MAC program. In alignment with the MAC protocol, participants were excluded if they had a current mental illness diagnoses as this type of clinical diagnosis can severely impair overall life functioning and performance. As such, increases in performance were considered of secondary priority and appropriate forms of support recommended. This information was obtained during the screening process and conversations with athletes about current psychological support.

The MAC protocol is inclusive of an initial session for case formulation and classification of participants as performance dysfunction or performance development (see Appendix). This classification system enables the instructor to ensure the participant has comprehended various aspects of the program before the program is progressed (Gardner \& Moore, 2007). The MAC program contains theory and exercises related to in-the-moment self-regulation, which is necessary for optimal competitive performance (Gardner \& Moore, 2004). Further, valued goal commitment exercises necessary for quality practice and long-term development of athletic skill are practiced by participants (Gardner \& Moore, 2004). Other topics included in the MAC program are decisionmaking, problem-solving, and behavioural processes and flexibility. The MAC program was delivered within an MI framework in each session. Although conversations with each participant varied, consistency was maintained by working within the spirit of MI, which emphasises partnership, acceptance, compassion and evocation (Miller \& Rollnick, 2012). For example, permission was sought from the athlete before completing experiential exercises tailored to their strengths and impairment, if required. MI was used to facilitate change by eliciting views on different topics (e.g., well-being and performance) and by exploring discrepancies between current states and desired states through open-ended questions, affirmations, (complex) reflections, and summaries (OARS; Miller \& Rollnick, 2012). If sustain talk (client responses that are consistent with maintaining the status quo) or ambivalence was encountered, OARS was repeated until engagement and change talk (client responses that are consistent with change) were evident. As per the MAC protocol, participants were given between session home activities (see Table 1).

\section{Procedure}

The eight-week program was delivered by one researcher (HM) who holds a teacher training certificate in mindfulness (2015), has been trained in acceptance and commitment therapy and MI (2015), and has delivered athlete career and education services to international athletes in Para and Olympic-Sports (2013 - 2018). The researcher (HM) was deemed proficient in MI through an analysis of a real-play session with an experienced MI practitioner using the Motivational Interviewing Treatment Integrity Coding Manual 4.2.1 (Moyers, Manuel, \& Ernst, 2015). The program was conducted through face-to-face individual sessions in meeting rooms at the VIS or at a convenient location for the participant with a private space (e.g., library meeting room). Although the majority of participants completed the weekly sessions consecutively, three participants required an extra week between sessions due to illness, competition, training schedules, or exams. Participants in the wait-list control group were instructed to continue with their typical activities, and were requested not to participate in other psychological skills programs. After the intervention, participants in the wait list control group were invited to complete the MAC program; no follow-up measures were conducted. 
Table 1: Outline of Individual Sessions

\begin{tabular}{|c|c|c|c|c|}
\hline Session & Session Aims & Psychoeducation & Experiential Activities & Between Session Exercise \\
\hline $\begin{array}{l}\text { 1. Assessment } \\
\text { interview }\end{array}$ & $\begin{array}{l}\text { Create comprehensive, } \\
\text { individualised athlete plan, \& } \\
\text { case formulation }\end{array}$ & $\begin{array}{l}\text { Exploration of athletes' } \\
\text { situation \& performance } \\
\text { classification }\end{array}$ & Baseline measurements, PRA & $\mathrm{n} / \mathrm{a}$ \\
\hline $\begin{array}{l}\text { 2. Introduction \& } \\
\text { Psycho-education }\end{array}$ & $\begin{array}{l}\text { Create understanding of key } \\
\text { program concepts, program } \\
\text { content, and build rapport }\end{array}$ & $\begin{array}{c}\text { Program theoretical rationale, } \\
\text { automatic self-regulation of } \\
\text { performance, 'ideal' } \\
\text { performance, poise }\end{array}$ & $\begin{array}{l}\text { BCE, ideal vs. non ideal } \\
\text { performance exercises }\end{array}$ & $\begin{array}{c}\text { BCE and "What I Have } \\
\text { Learned" exercises (repeats for } \\
\text { each session) }\end{array}$ \\
\hline 3. Mindfulness & $\begin{array}{l}\text { RPS, use mindfulness to } \\
\text { improve performance }\end{array}$ & $\begin{array}{l}\text { Mindfulness, cognitive fusion } \\
\text { and defusion }\end{array}$ & $\begin{array}{l}\text { Mindful eating, BCE, mindful } \\
\text { vs. mindless }\end{array}$ & $\begin{array}{c}\text { “Washing a Dish" mindfulness } \\
\text { exercise }\end{array}$ \\
\hline $\begin{array}{l}\text { 4. Values \& Values- } \\
\text { Driven Behaviour }\end{array}$ & $\begin{array}{l}\text { RPS, differentiate values and } \\
\text { goals }\end{array}$ & $\begin{array}{l}\text { Values, goals, value-driven / } \\
\text { emotion-driven behaviour, } \\
\text { and function of emotions }\end{array}$ & $\begin{array}{c}\text { BCE, MoB exercise, } \\
\text { performance and life values } \\
\text { card sort identification }\end{array}$ & $\begin{array}{c}\text { "Performance Values"/ "Given } \\
\text { Up for Emotions"/ Mindful } \\
\text { exercise MoB }\end{array}$ \\
\hline 5. Acceptance & $\begin{array}{l}\text { RPS, identify experiential } \\
\text { avoidance strategies, apply } \\
\text { experiential acceptance }\end{array}$ & $\begin{array}{l}\text { Experiential acceptance and } \\
\text { experiential avoidance }\end{array}$ & $\begin{array}{c}\text { MoB/BCE, LoS/“Pushing the } \\
\text { Paper Away”/“Joining the } \\
\text { DOTS” exercise }\end{array}$ & $\begin{array}{l}\text { LoS, mindfulness training, } \\
\text { “Emotion \& Performance } \\
\text { Interference" exercise }\end{array}$ \\
\hline $\begin{array}{l}\text { 6. Enhancing } \\
\text { Commitment }\end{array}$ & $\begin{array}{l}\text { RPS, apply growth mindset to } \\
\text { performance, gain } \\
\text { commitment to performance }\end{array}$ & $\begin{array}{l}\text { Commitment, motivation, } \\
\text { fixed and growth mindsets }\end{array}$ & $\begin{array}{l}\text { Extension of BCE, Body Scan, } \\
\text { resiliency formula \& reframe } \\
\text { exercise. }\end{array}$ & $\begin{array}{l}\text { Body Scan, mindfulness in } \\
\text { competition, 'Committing to } \\
\text { Performance Values' exercise }\end{array}$ \\
\hline $\begin{array}{c}\text { 7. Skill } \\
\text { Consolidation }\end{array}$ & $\begin{array}{l}\text { RPS, task-focused attention, } \\
\text { connect breath, mind, body }\end{array}$ & $\begin{array}{l}\text { Physiology of the breath, task- } \\
\text { focused attention }\end{array}$ & $\begin{array}{l}\text { Extensions of MoB, BCE, task- } \\
\text { focused attention exercise. }\end{array}$ & $\begin{array}{l}\text { Task-focused attention \& } \\
\text { reflection/mindfulness exercise }\end{array}$ \\
\hline $\begin{array}{l}\text { 8. Maintaining \& } \\
\text { Enhancing MAC }\end{array}$ & $\begin{array}{c}\text { Program reflection, identify } \\
\text { improvement, gain future } \\
\text { commitment }\end{array}$ & $\begin{array}{c}\text { Combing all concepts, link } \\
\text { between reflection and self- } \\
\text { awareness }\end{array}$ & $\begin{array}{l}\text { Athlete "instructor" for } \\
\text { mindfulness, reflection, Post- } \\
\text { MAC planning, PRA }\end{array}$ & MAC skills \\
\hline
\end{tabular}

2 Note. PRA = performance rating activity; $\mathrm{BCE}=$ brief centring exercise; RPS = review previous session; $\mathrm{LoS}=$ leaves on stream; MoB = mindfulness of breath. 


\section{Outcome Measurements}

Participants were assessed at baseline (Week 0 ) and immediately after the intervention (Week 8) using an online survey (Qualtrics).

\section{Primary outcomes.}

Subjective well-being (hedonic tradition). The Scale of Positive and Negative Experience (SPANE; Diener et al., 2010) was used to measure the affective component of subjective-well-being across the dimensions of positive and negative affect (e.g., "happy" and "sad"; Diener et al., 2010). Participants reported how much they experienced each feeling during the past four weeks across twelve items on a 5 -point scale $(1=$ very rarely or never to $5=$ very often or always; a balance-score is created by combining the two dimensions). The SPANE has consistently performed well with respect to reliability and convergent validity with other measures of emotion, well-being, and life satisfaction (Diener at al., 2010).

The Satisfaction with Life Scale (Diener, Emmons, Larsen, \& Griffin, 1985) was used to measure the cognitive component of subjective well-being. Participants rated their agreement across five items (e.g., "I am satisfied with my life") on a 7-point scale ( $1=$ strongly disagree to $7=$ strongly agree). The scale has good psychometric properties with regard to reliability, validity and temporal stability (Pavot \& Diener, 1993).

Psychological well-being (Eudaimonic tradition). Ryff's (1989) 42-item Psychological WellBeing Scale was used to assess self-acceptance, environmental mastery, personal growth, autonomy, positive relation with others and purpose in life (e.g., "I have a sense of direction and purpose in life"). Participants rated items on a 6-point scale $(1=$ disagree to $6=$ agree). Responses to negatively scored items were reversed for scoring. In the initial validation study, the scale had high internal consistency, test-retest reliability, and convergent and discriminant validity with other measures (Ryff et al., 2012).

Social well-being (Eudaimonic tradition). Keyes's (1998) 15-item Social Well-Being Questionnaire was used to evaluate social acceptance, actualisation, coherence, integration, and contribution using a 7 -point scale $(1=$ strongly disagree to $7=$ strongly agree $)$. The scale has good construct validity and internal consistency (Keyes \& Shapiro, 2004).

\section{Secondary outcomes.}

Physical well-being/health. Similar to Reinboth and Duda (2004), the 15-item Athlete Burnout Measure (ABM; Raedeke \& Smith, 2001) was used to assess athletes experience of emotional and lack of physical well-being in their sport. The ABM contains three subscales: Emotional/Physical Exhaustion, Reduced Sense of Accomplishment, and Sport Devaluation. Participants responded to items on a 5 -point scale $(1=$ almost never to $5=$ almost always $)$. The scale has good convergent and discriminant validity (Cresswell \& Eklund, 2006), and internal consistency (Raedeke \& Smith, 2001).

The Pain Catastrophising Scale (Sullivan, Bishop, \& Pivik, 1995) is a 13-item measure with three subscales-Rumination, Magnification, and Helplessness - that contribute to heightened levels of pain and emotional distress. It uses a 5-point scale $(0=$ not at all to $4=$ all of the time $)$. The scale has good criterion-related validity, internal consistency (Osman et al., 2000), and test-retest reliability (Sullivan et al., 1995).

Experiential avoidance/acceptance. The Acceptance and Action Questionnaire-II (Bond et al., $2011)$ is a 10-item measure of psychological flexibility and acceptance, and uses a 7-point scale $(1=$ never true to $7=$ always true). The scale has satisfactory validity and reliability (Fledderus, Oude Voshaar, Ten Klooster, \& Bohlmeijer, 2012).

Mindfulness. The 12-item Cognitive and Affective Mindfulness Scale-Revised (Feldman, Hayes, Kumar, Greeson, \& Laurenceau, 2007) was used to measure mindful approaches to internal experiences using a 4-point scale $(1=$ rarely/not at all to $4=$ almost always). Participants were asked 
to consider the items in the context of their sport. The scale has acceptable internal consistency (Baer et al., 2006) and good construct validity (Feldman et al., 2007).

\section{Data Analysis}

SPSS Version 23 was used to analyse data. Data were checked for normality using the Shapiro Wilk test, normal Q-Q plot, and we found acceptable values of skewness and kurtosis $( \pm 2$ and 5 respectively, Kendall \& Stuart, 1958), followed by reliability analyses. To avoid bias and maximise the randomisation process, intention-to-treat analysis was used. All outcomes were analysed using Analysis of Covariance (ANCOVA) of the post-intervention scores with baseline scores used as covariates, and the mean difference between groups and associated $95 \%$ confidence intervals were calculated. Being a pilot study, this trial was not adequately powered to detect statistically significant between-group differences. Consequently, partial eta squared was calculated for each of the study outcomes to provide a measure of effect size (i.e., practical significance). Effect sizes were interpreted according to Cohen (1988) where an effect of $\eta 2<.01$ was regarded as small, $\eta 2=.06$ represents a medium effect, and if $\eta 2>.14$, it was considered a large effect.

\section{Results}

\section{Flow of Participants Through the Trial and MAC Performance Classification}

The intervention was delivered as intended and the flow of participants, with reasons for exclusion, can be seen in Figure 2. No adverse events were reported during the trial. Participant

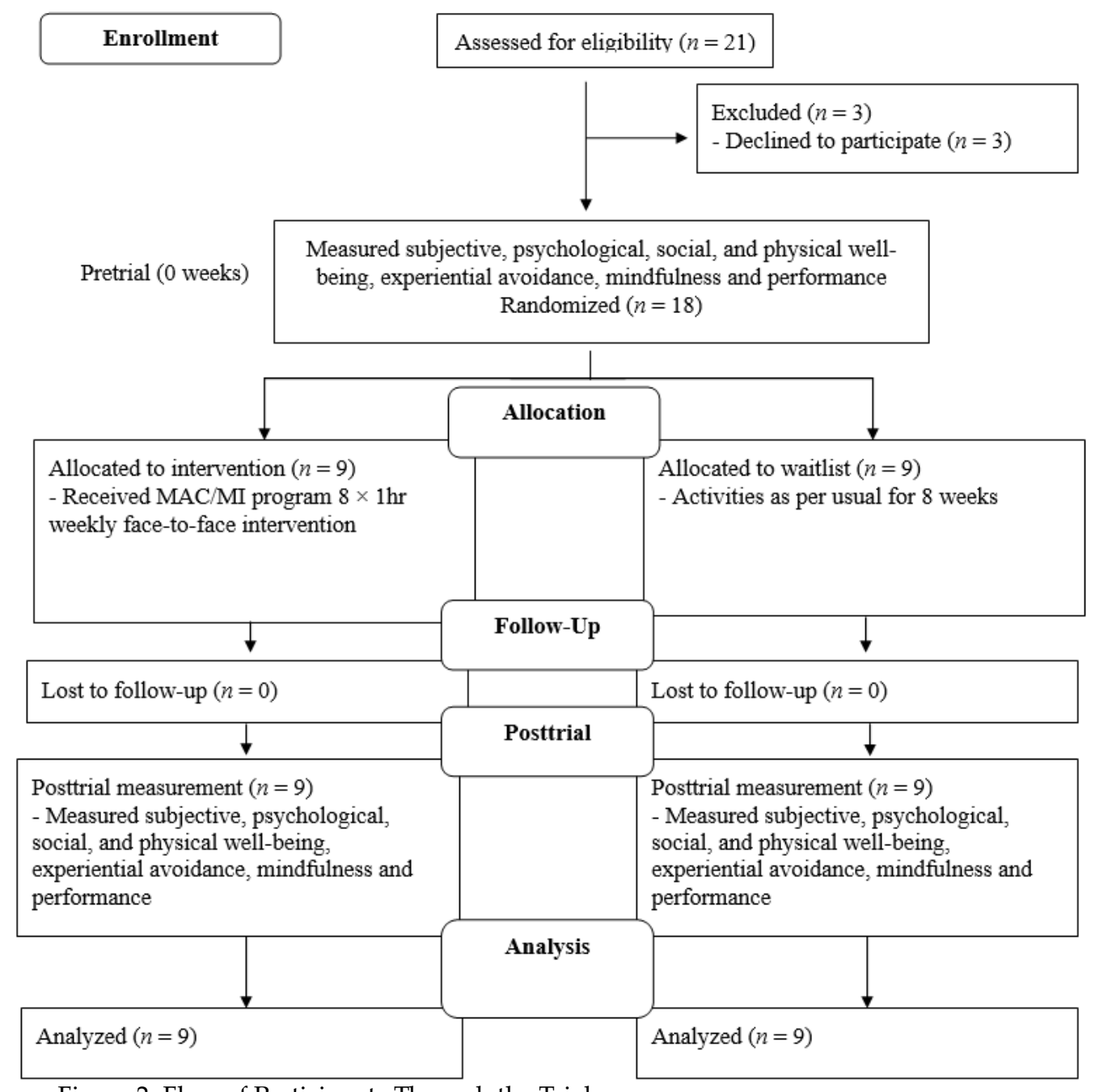

Figure 2. Flow of Participants Through the Trial 
characteristics are shown in Table 2. The only difference between groups was in individual or team sport classification.

Using the MAC protocol (Gardner \& Moore, 2004), two participants were classified as performance dysfunction and seven as performance development (see Appendix for classification procedure). Participants with the classification of performance dysfunction completed longer sessions each week to ensure key concepts were fully understood and any problems related to performance could be addressed within each session and understood from a mindfulness, acceptance, or commitment lens.

\section{Potential Efficacy of Intervention on Primary Outcomes}

Adjusted between-group differences (and effect sizes) for the primary study outcomes are presented in Table 3. Differences in all domains of subjective well-being (H1a) were identified as having favoured the intervention group, with moderate to large effect sizes (i.e., $\eta 2$ range $=.06$ to .29 ). Statistical significance was discerned within 'satisfaction with life' (mean [95\%CI's] $=5.5$ [.8 to 10.2]). Similarly, between-group differences for all dimensions of psychological well-being ( $\mathrm{H} 1 \mathrm{~b})$, as determined by the researchers from the results, favoured the intervention group, with moderate to large effect sizes ( $\eta 2$ range $=.05$ to .28). Differences were significant for 'personal growth' (mean difference $\left[95 \% \mathrm{CI}^{\prime} \mathrm{s}\right]=2.6[.3$ to 4.9$]$ ) and 'positive relations' (mean difference $\left[95 \% \mathrm{CI}^{\prime}\right.$ 's] $=3.5[.2$ to 6.9]). No meaningful intervention effects were found for dimensions of social well-being ( $\eta 2$ range = .00 to $.08 ; \mathrm{H} 1 \mathrm{c})$.

Table 2: Demographics of Participants

\begin{tabular}{|c|c|c|}
\hline \multirow[b]{2}{*}{ Characteristic } & \multicolumn{2}{|c|}{ Randomised $(N=18)$} \\
\hline & Intervention $(n=9)$ & Waitlist Control $(n=9)$ \\
\hline Age (yrs.), mean $(S D)$ & $34(12)$ & $31(12)$ \\
\hline Gender, $n$ males $(\%)$ & $2(22 \%)$ & $3(33 \%)$ \\
\hline \multicolumn{3}{|l|}{ Type of sport } \\
\hline $\begin{array}{l}\text { Individual (e.g., athletics, } \\
\text { cycling, \& swimming) }\end{array}$ & $8(89 \%)$ & $6(67 \%)$ \\
\hline Team & $1(11 \%)$ & $3(33 \%)$ \\
\hline \multicolumn{3}{|l|}{$\begin{array}{c}\text { Sport (e.g., wheelchair } \\
\text { basketball \& rugby) } \\
\text { Level }\end{array}$} \\
\hline National & 0 & $1(11 \%)$ \\
\hline International & $4(44 \%)$ & $4(44 \%)$ \\
\hline Paralympic & $5(56 \%)$ & $4(44 \%)$ \\
\hline \multicolumn{3}{|l|}{ Nature of impairment } \\
\hline Acquired & $6(67 \%)$ & $6(67 \%)$ \\
\hline Congenital & $3(33 \%)$ & $3(33 \%)$ \\
\hline \multicolumn{3}{|l|}{ Elite sport career duration } \\
\hline Less than $2 \mathrm{yr}$. & $2(22 \%)$ & $2(22 \%)$ \\
\hline $2-5 \mathrm{yr}$. & $3(33 \%)$ & $3(33 \%)$ \\
\hline $5-10 \mathrm{yr}$. & $3(33 \%)$ & $3(33 \%)$ \\
\hline $10+\mathrm{yr}$. & $1(11 \%)$ & $1(11 \%)$ \\
\hline
\end{tabular}

\section{Potential Efficacy of Intervention on Secondary Outcomes}

Adjusted between-group differences (and effect sizes) for secondary outcomes are presented in Table 4. Regarding physical well-being (H3), statistically significant effects were observed for pain catastrophising scale total score (mean difference $[95 \% \mathrm{CI}]=-5.8[-11.3$ to -.3$]$ ) and the 'helplessness' subscale (mean difference [ $\left.95 \% \mathrm{CI}^{\prime} \mathrm{s}\right]=-3.0[-5.5$ to -0.5$]$ ). Although a large effect size was also found for the pain subscale of 'magnification', the Cronbach's alpha coefficient did not reach an acceptable 
level (e.g., <.49). Group differences in all remaining secondary outcomes (H2a and H2b) were not significant and tended to be small in magnitude.

\section{Discussion}

We have provided preliminary support through a pilot RCT for the use of the MAC program delivered within an MI framework to improve the perceived well-being of athletes in Para Sports. The results partially confirms our hypotheses. When compared to the wait list control group, participants in the intervention group reported a significant increase in several dimensions of wellbeing associated with primary outcomes and hypothesis - satisfaction with life (H1a, hedonia), positive relation with others, and personal growth ( $\mathrm{H} 1 \mathrm{~b}$, eudaimonia). Participants in the intervention group also reported significant improvements in the secondary outcome pain catastrophising perceptions (helplessness and total scale score; H3, physical well-being / health), when compared to the wait list control group. Supporting our hypotheses was also the general consistency in the pattern of effects for our primary outcomes. For example, when statistically significant findings were seen for sub-dimensions of subjective and psychological well-being, the remaining (non-significant) subdomains typically showed moderate to large effect sizes in the same direction. However, our first hypothesis was not entirely supported due to a lack of improvement in social well-being (H1c; eudaimonia), and our secondary hypothesis was not supported due to a lack of significant improvement in mindfulness $(\mathrm{H} 2 \mathrm{a})$ and acceptance $(\mathrm{H} 2 \mathrm{~b})$. Although not reaching significance, the trend for improvement in mindfulness was reflective of other MAC interventions (e.g., Goodman et al., 2014), and has the potential to suggest the relevancy of mindfulness for athletes in Para Sports. The negligible changes in pre to post scores for acceptance, the other main cognitive approach in the MAC program, was potentially indicative of established higher levels of acceptance for athletes in Para Sports.

Despite a lack of full support for our hypotheses, the results are encouraging for a number of reasons. The trial compliments the growing body of evidence supporting the MAC program to improve athlete well-being (e.g., Bernier et al., 2009; Gardner \& Moore, 2004) through providing large effect sizes for multiple domains of primary and secondary outcomes. Importantly, the results from primary outcomes have been underpinned by the traditions of hedonia and eudaimonia, with athletes in the intervention group reporting well-being improvements in domains associated with both theoretical traditions. Further, it is the first intervention to combine the MAC protocol within an MI framework, thereby providing an increased specificity for how the program was delivered. Although we cannot ascertain which aspects of the program (MAC, MI or both) contributed to the outcomes, it has been suggested that combining MI with other interventions can help to increase adherence, promote synergistic effects, and lead to better outcomes (Csillik, 2015; Nar-King, Earnshaw, \& Breckon, 2013). It is possible that the MI framework served to increase intrinsic motivation for change (Csillik, 2015), and complemented the value- and strength-driven behaviour component of the MAC program.

The results of this study also reflect the improvements in subjective and psychological wellbeing in other populations (e.g. chronic pain patients) after mindfulness and/or acceptancecommitment therapy (Grossman et al., 2004; Johnston, Foster, Shennan, Starkey, \& Johnson, 2010). Acceptance of pain, and engaging in activities despite pain, have been found to correlate positively with quality of life (Mason, Mathias, \& Skevington, 2008) and align with the reported improvements in satisfaction with life and reduction in pain catastrophising. 
Table 3: Mean (SD) of Groups, Mean (SD) Difference Within and Between (i.e., Difference-in-Difference) Groups (95\% CI), and Alpha Coefficients for Primary Hypothesis Outcomes

\begin{tabular}{|c|c|c|c|c|c|c|c|c|c|}
\hline \multirow[b]{3}{*}{ Outcome } & \multicolumn{4}{|c|}{ Groups } & \multirow{2}{*}{\multicolumn{2}{|c|}{$\begin{array}{c}\text { Difference Within } \\
\text { Groups } \\
\text { Post Minus Pre } \\
\end{array}$}} & \multirow{3}{*}{$\begin{array}{c}\text { Difference Between } \\
\text { Groups } \\
\text { Post Minus Pre } \\
\text { Int Minus } \\
\text { Con }^{\wedge}\end{array}$} & \multirow{3}{*}{$\begin{array}{l}\text { Partial } \\
\text { eta } \\
\text { squared }\end{array}$} & \multirow[b]{3}{*}{$\alpha$} \\
\hline & \multicolumn{2}{|c|}{ Pre } & \multicolumn{2}{|c|}{ Post } & & & & & \\
\hline & Int & Con & Int & Con & Int & Con & & & \\
\hline \multicolumn{10}{|l|}{ Subjective Well-Being } \\
\hline Satisfaction with life & $24.2(6.1)$ & $23.4(4.8)$ & $29.0(5.4)$ & $23.0(6.0)$ & $4.8(5.6)$ & $-.4(4.3)$ & $5.5^{*}[.8,10.2]$ & .29 & .78 \\
\hline Positive emotions & $19.2(2.1)$ & $18.7(1.9)$ & $25.3(4.2)$ & $21.1(4.4)$ & $6.1(2.8)$ & $2.4(5.3)$ & $3.9[-.4,8.3]$ & .20 & .73 \\
\hline Negative emotions & $14.2(2.1)$ & $16.3(3.6)$ & $13.6(3.3)$ & $16.8(5.5)$ & $-.7(3.2)$ & $.4(3.8)$ & $-1.2[-5.1-2.7]$ & .06 & .66 \\
\hline Balance of emotions & $5.0(3.6)$ & $2.3(4.5)$ & $11.8(6.5)$ & $4.3(8.6)$ & $6.7(4.8)$ & $2.0(8.5)$ & $5.2[-2.2,12.8]$ & .15 & .77 \\
\hline Psychological Well-Being & & & & & & & & & .93 \\
\hline Autonomy & $28.1(5.4)$ & $29.7(5.5)$ & $31.3(3.4)$ & $29.7(5.5)$ & $3.2(3.6)$ & $0.0(2.6)$ & $2.7^{* *}[.0,5.5]$ & .23 & .72 \\
\hline Environmental mastery & $29.8(6.3)$ & $32.2(4.6)$ & $34.3(5.5)$ & $32.4(6.1)$ & $4.6(6.6)$ & $.2(3.5)$ & $3.4[-1.8,8.5]$ & .11 & .75 \\
\hline Personal growth & $36.9(4.7)$ & $38.7(3.0)$ & $40.0(2.4)$ & $38.2(3.3)$ & $3.1(3.3)$ & $-.4(2.7)$ & $2.6^{*}[.3,4.9]$ & .28 & .80 \\
\hline Positive relations & $34.7(5.1)$ & $36.9(5.7)$ & $36.4(6.0)$ & $35.2(6.8)$ & $1.8(3.4)$ & $-1.7(2.9)$ & $3.5^{*}[.19,6.9]$ & .25 & .80 \\
\hline Purpose in life & $34.3(6.7)$ & $35.0(4.6)$ & $36.8(2.9)$ & $35.8(4.2)$ & $2.4(5.3)$ & $.8(3.3)$ & $1.3[-1.7,4.2]$ & .05 & .79 \\
\hline Self-acceptance & $32.7(5.0)$ & $32.3(5.6)$ & $35.3(6.1)$ & $32.7(5.5)$ & $2.7(4.6)$ & $.3(3.2)$ & $2.4[-1.6,6.4]$ & .10 & .71 \\
\hline Social Well-Being & & & & & & & & & .74 \\
\hline Acceptance & $13.7(2.4)$ & $14.7(3.9)$ & $13.4(2.0)$ & $14.0(3.0)$ & $-.2(2.3)$ & $-.7(3.2)$ & $1.0[-1.5,3.5]$ & .05 & .51 \\
\hline Actualisation & $14.0(2.6)$ & $15.9(3.2)$ & $14.6(3.2)$ & $17.1(2.9)$ & $.6(3.2)$ & $1.2(2.4)$ & $-1.5[-4.2,1.3]$ & .08 & .56 \\
\hline Coherence & $13.2(2.5)$ & $14.3(3.5)$ & 13.1(3.9) & $14.2(2.7)$ & $-.1(4.2)$ & $-.1(2.4)$ & $-.5[-3.5,2.6]$ & .01 & .41 \\
\hline Contribution & $17.3(3.5)$ & $18.2(3.6)$ & $17.7(3.4)$ & $18.3(3.5)$ & $.3(2.4)$ & $.1(2.6)$ & $-.0[-2.4,2.4]$ & .00 & .77 \\
\hline Integration & $17.3(2.8)$ & $16.8(3.3)$ & $17.8(2.6)$ & $16.9(4.0)$ & $.4(2.3)$ & $.1(4.3)$ & $-.4[-2.7,1.9]$ & .01 & .64 \\
\hline
\end{tabular}

Note. $\quad$ Int $=$ intervention group, Con $=$ wait list control group. ${ }^{\wedge}$ Based on ANCOVA adjusted means. ${ }^{*} p<.05,{ }^{* *} p=0.05$. Table 3 displays the average scores for secondary outcomes at pre and post time points for both intervention and wait-list control groups. These scores were subsequently used to calculate differences within groups, by comparing pre and post

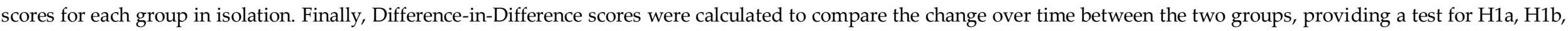

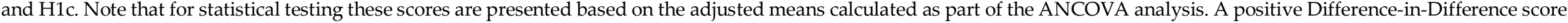
represents that scores in the intervention group changed to a higher degree than in the wait-list control group, while a negative score represents the opposite result. 
Table 4: Mean (SD) of Groups, Mean (SD) Difference Within and Between Groups (95\% CI), and Alpha Coefficients for Outcomes of Hypothesis Two and Three

\begin{tabular}{|c|c|c|c|c|c|c|c|c|c|}
\hline \multirow[t]{3}{*}{ Outcome } & \multicolumn{4}{|c|}{ Groups } & \multirow{2}{*}{\multicolumn{2}{|c|}{$\begin{array}{c}\text { Difference Within } \\
\text { Groups } \\
\text { Post Minus Pre }\end{array}$}} & \multirow{3}{*}{$\begin{array}{c}\text { Difference Between } \\
\text { Groups } \\
\text { Post Minus Pre } \\
\text { Int -Con }^{\wedge} \\
\end{array}$} & \multirow{3}{*}{$\begin{array}{c}\text { Partial eta } \\
\text { squared }\end{array}$} & \multirow[b]{3}{*}{$\alpha$} \\
\hline & \multicolumn{2}{|c|}{ Pre } & \multicolumn{2}{|c|}{ Post } & & & & & \\
\hline & Int & Con & Int & Con & Int & Con & & & \\
\hline Mindfulness & $30.9(6.6)$ & $32.8(6.2)$ & $34.1(5.7)$ & $33.9(5.5)$ & $3.2(4.0)$ & $1.1(4.7)$ & $1.5[-2.5,5.4]$ & .04 & .87 \\
\hline Experiential Acceptance & $34.7(3.3)$ & $33.8(5.9)$ & $34.8(3.0)$ & $35.1(3.9)$ & $.1(2.9)$ & $1.3(3.6)$ & $-.8[-3.26,1.66]$ & .03 & .87 \\
\hline Performance & & & & & & & & & .83 \\
\hline Concentration & $3.4(.9)$ & $3.9(.9)$ & $3.9(.9)$ & $3.6(.9)$ & $.4(1.1)$ & $-.3(1.2)$ & $.4[-.6,1.4]$ & .05 & \\
\hline Strength & $3.7(1.0)$ & $4.2(1.1)$ & $4.1(.8)$ & $3.8(1.2)$ & $.4(1.0)$ & $-.4(.9)$ & $.6[-.3,1.5]$ & .14 & \\
\hline Motivation & $3.6(1.1)$ & $4.1(.8)$ & $4.0(.9)$ & $3.9(1.1)$ & $.4(.9)$ & $-.2(1.2)$ & $.3[-.6,1.3]$ & 04 & \\
\hline Quickness & $2.9(.9)$ & $3.7(1.2)$ & $4.0(1.0)$ & $3.4(1.0)$ & $1.1(1.4)$ & $-.2(.7)$ & $.9[-.1,1.9]$ & .20 & \\
\hline Fitness & $3.9(.6)$ & $4.6(.7)$ & $4.1(.8)$ & $4.1(.6)$ & $.2(.7)$ & $-.4(.5)$ & $.4[-.2,1.1]$ & .12 & \\
\hline Endurance & $3.9(.9)$ & $4.1(.9)$ & $4.1(.8)$ & $3.9(.9)$ & $.2(.1)$ & $-.2(1.0)$ & $.3[-.6,1.1]$ & .03 & \\
\hline Mechanics & $3.8(.7)$ & $3.4(1.1)$ & $3.7(.9)$ & $3.4(1.0)$ & $-.1(.9)$ & $0.0(.5)$ & $-.0[-.7, .7]$ & .00 & \\
\hline Aggressiveness & $3.6(1.1)$ & $3.7(1.0)$ & $3.7(.9)$ & $3.9(.8)$ & $.1(.6)$ & $2(1.0)$ & $-.2[-.8, .5]$ & .02 & \\
\hline Agility & $3.1(.9)$ & $3.7(1.2)$ & $4.0(.7)$ & $3.3(1.0)$ & $.9(1.2)$ & $-.3(.7)$ & $.9^{*}[.1,1.7]$ & .28 & \\
\hline Team cohesion & $3.9(.8)$ & $3.8(.8)$ & $4.0(.5)$ & $3.6(.7)$ & $.1(.6)$ & $-.2(.7)$ & $.4[-.1, .9]$ & .16 & \\
\hline Overall performance & $3.9(.6)$ & $3.9(.9)$ & $4.3(.7)$ & $3.9(.8)$ & $.4(.7)$ & $0.0(1.0)$ & $.4[-.23,1.2]$ & .10 & \\
\hline Physical Well-Being & & & & & & & & & \\
\hline Pain (total scale) & $13.2(6.1)$ & $16.3(11.7)$ & $7.0(3.8)$ & $13.6(7.2)$ & $-6.2(5.1)$ & $-2.8(11.3)$ & $-5.8^{*}[-11.3,-.3]$ & .25 & .92 \\
\hline Rumination & $5.2(2.5)$ & $5.6(4.3)$ & $3.1(2.1)$ & $4.4(3.4)$ & $-2.1(2.3)$ & $-1.1(3.6)$ & $-1.2[-3.6,1.3]$ & .07 & .85 \\
\hline Magnification & $3.0(1.7)$ & $3.6(2.8)$ & $1.6(1.8)$ & $3.6(1.9)$ & $-1.4(1.9)$ & $0.0(2.2)$ & $-1.77[-3.4,-.1]$ & .26 & .49 \\
\hline Helplessness & $5.0(3.1)$ & $7.2(5.3)$ & $2.3(1.2)$ & $5.6(3.1)$ & $-2.7(2.7)$ & $-1.7(5.7)$ & $-3.0^{*}[-5.5,-.5]$ & .30 & .88 \\
\hline E/P exhaustion & $15.3(3.9)$ & $12.4(5.0)$ & $11.8(3.9)$ & $12.1(2.9)$ & $-3.6(5.1)$ & $-1.0(2.5)$ & $-1.1[-4.7,2.4]$ & .03 & .84 \\
\hline Reduced accomp. & $12.8(4.9)$ & $12.1(3.8)$ & $11.2(4.2)$ & $11.1(3.4)$ & $-1.6(5.6)$ & $.3(2.5)$ & $-.2[-3.7,3.4]$ & .01 & .84 \\
\hline Sport devaluation & $10.9(5.1)$ & & $9.6(4.7)$ & $9.7(3.1)$ & $-1.3(2.8)$ & & $-1.3[-3.9,1.3]$ & .07 & .83 \\
\hline
\end{tabular}

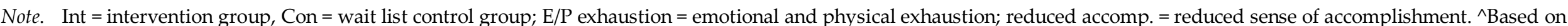
ANCOVA adjusted means. ${ }^{*} p<.05$. Please see Table 3 for a detailed explanation of the calculations used in Table 4. 
Thus, the current study has helped to address some of the well-being needs identified in previous research focused upon athletes in Para Sports (Macdougall et al., 2016). It was interesting that there were no significant improvements for the primary outcome of social well-being for the intervention group when compared to the control group. One explanation is the application of Keye's (1998) Social Well-Being Scale. Although some of these dimensions have been shown to be relevant to athletes in Para Sports (e.g., social contribution, Macdougall et al., 2016), it is possible that other aspects of social well-being not included in the scale are more applicable (e.g., social connections). Further, the intervention was one-to-one as opposed to a group format, and did not focus on social well-being, expressing gratitude, or giving. Whereas the individualised sessions permitted for a tailored approach, a group format may be more conducive to social well-being enhancement, and warrants further investigation. Especially as contributing to communities has been previously identified as a well-being strength for athletes in Para Sports (Macdougall et al., 2016).

With respect to the encouraging results of the trial, the predicted theoretical mechanisms of mindfulness and acceptance-based interventions, and neuroplasticity (Gardner \& Moore, 2012), are useful to consider. Mindfulness and acceptance-based interventions may promote enhanced mental efficiency as a result of developing greater awareness and acceptance of internal experiences (e.g., stress; Gardner \& Moore, 2012). Through the MAC program, participants systematically repeat exercises of attentional self-regulation and are able to detect and direct their attentional focus to a desired stimulus (e.g., their performance demands) without needing to control other relevant sensory or internal bodily data. Given that MI has been a well-supported approach for increasing intrinsic motivation (Csillik, 2015), it may also serve to increase motivation for dedicated and continuous mental practice in sport. However, the pilot nature of the study and thus potentially the resultant lack of improvement in mindfulness and acceptance, means that no firm conclusions can be drawn as to the effectiveness of MAC program components within an MI framework.

The main strength of this trial was its design. Another strength of the trial was the tailored approach that was achieved through the classification of participants as 'performance development' or 'performance dysfunction' based on initial case formulations, as well as within individual sessions. The ability to tailor sessions to participants is an important aspect of programs (Bernier et al., 2009) and may have mitigated any risks associated with applying a program developed for athletes in Olympic Sports. For example, body scan exercises were adapted to suit the nature of athletes' physical impairments as well as preferences when focusing on non-functional limbs. When compared to previous research using the MAC intervention (e.g., Goodman et al., 2014), the current trial was distinctive through primary outcome measures aligned with hedonia and eudaimonia. As such, the current trial has been clear in the theoretical traditions used to define well-being within the current study, and addresses some of the shortcomings of sport psychology research identified within the review by Lundqvist (2011).

There were two deviations from the registered trial protocol: data related to sport-specific wellbeing, performance, and qualitative data were collected and will be reported in a subsequent paper. The second deviation was that social well-being was adjusted to be included as a primary outcome rather than secondary. This decision was based on further analysis of well-being frameworks within the sport context (e.g., Lundqvist \& Raglin, 2015) after trial registration. Further, the current trial was limited by the small sample size of 18 participants who were at various stages of their seasonal preparation. As such, the effects of the intervention were required to be very large to detect significant changes as a result of the program and potentially minimised themes from being observed. Although we recognise this as a limitation, it must be considered in the context of the study population. For example, elite athletes in Para Sports represent a very small proportion of the population, and have competing demands on their time making large-scale recruitment difficult. Also, given what is at stake for athlete participants, any attempts at intervening with a larger group (i.e., in a powered, nonpilot trial) should apply evidence-based interventions that have been piloted and have empirical support. Thus, the small sample size was expected and future research with a larger sample will be able to confirm or refute our findings. Specifically, larger samples will be able to confirm the 
relevancy of mindfulness and acceptance for athletes in Para Sports and serve as a validity check for the MAC intervention within an MI framework for this population.

Another limitation included the inability to determine which component of the program brought about the changes observed - the MAC program or the MI framework. Lastly, self-reported outcome measures were used, no follow-up measures were conducted, participants were not blinded to the intervention, and desirability bias may have exaggerated the intervention effects. It is recommended that larger scale trials with subjective and objective measures of well-being and performance, and three and six-month follow-up be completed. These future trials need to include athletes in Para Sports with different impairments (e.g., sensory impairments) before this type of intervention could be considered generalisable to the wider athlete community. Future trials should also attempt to measure the relative contributions of MAC compared to MI in order to determine the effectiveness of each and value of combining positive psychology interventions. These trials could also consolidate or refute the relevancy of MAC for hedonia and eudaomonia, their respective dimensions and measures, inclusive of physical health and well-being. Lastly, it is suggested that data related to the timing of intervention delivery during the season, and the possible impacts this has on the intervention is explored. For example, does adherence and effect sizes increase if the intervention is delivered during the off-season or pre-season?

We have presented preliminary evidence that an eight-module MAC program combined with an MI framework is one way to enhance perceived performance and well-being of athletes in Para Sports in a relatively short period of time. Thus, athletes in Para Sports may find that the use of mindfulness, acceptance, and commitment skills can provide benefit to their lives on and off the sporting field.

\section{Perspectives}

The results of this study indicate preliminary usefulness of targeted psychological interventions for athletes in Para Sport with physical impairments. The reported improvements across multiple well-being dimensions need to be considered within the context of the pilot nature of the study and small sample size. However, these findings are important in an era where well-being and mindfulness have been linked to performance (Bernier et al., 2009; Martin, 2012), and athletes perform in an environment linked extrinsically to gold medal performance and funding. Small and financially inexpensive interventions, such as those used in this RCT, provide clarity around how athletes may potentially operate and function in this type of environment, and provide sport practitioners with the how of athlete support via specific skills and tools.

\section{Author Affiliations:}

1 Department of Management; Faculty of Business, Economics and Law; College of Arts, Social Sciences and Commerce; Centre for Sport and Social Impact, LaTrobe University, Bundoora, VIC, 3086;

h.macdougall@latrobe.edu.au;

2 Faculty of Health Sciences; College of Science, Health and Engineering; Department of Public Health, LaTrobe University, Bundoora; paul.ohalloran@latrobe.edu.au

3 Swinburne Business School, Faculty of Business and Law, Swinburne University, Hawthorn; e.sherry@swin.edu.au

4 School of Allied Health; College of Science, Health and Engineering; La Trobe University, Bundoora, VIC; n.shields@latrobe.edu.au

* Corresponding author: Hannah Macdougall email: h.macdougall@latrobe.edu.au

Author Contributions: Conceptualization, HM, PE, ES \& NS; Methodology, HM, PE, ES \& NS; Writing-Original Draft Preparation, HM, PE, ES \& NS; Writing-Review \& Editing, HM, PE, ES \& NS.

Funding: This research received no external funding.

Conflicts of Interest: The authors declare no conflict of interest.

\section{References}

Baer, R. A., Smith, G. T., Hopkins, J., Krietemeyer, J., \& Toney, L. (2006). Using self-report assessment methods to explore facets of mindfulness. Assessment, 13, 27-45. doi:10.1177/1073191105283504 
Bernier, M., Thienot, E., Codron, R., \& Fournier, J. F. (2009). Mindfulness and acceptance approaches in sport performance. Journal of Clinical Sport Psychology, 3, 320-333. doi:10.1123/jcsp.3.4.320

Bond, F. W., Hayes, S. C., Baer, R. A., Carpenter, K. M., Guenole, N., Orcutt, H. K., ... \& Zettle, R. D. (2011). Preliminary psychometric properties of the Acceptance and Action Questionnaire-II: A revised measure of psychological inflexibility and experiential avoidance. BehaviorTherapy, 42(4), 676-688.

Bricker, J. B., \& Tollison, S. J. (2011). Comparison of motivational interviewing with acceptance and commitment therapy: A conceptual and clinical review. Behavioral and Cognitive Psychotherapy, 39, 541-559. doi:10.1017/S1352465810000901.

Brown, K.W., \& Ryan, R.M. (2003). The benefits of being present: Mindfulness and its role in psychological wellbeing. Journal of Personality and Social Psychology, 84, 822-848. doi:10.1037/0022-3514.84.4.822

Campbell, E. \& Jones, G. (2002). Sources of stress experienced by elite male wheelchair basketball players. Adapted Physical Activity Quarterly, 19, 82-99. doi: 10.1123/apaq.19.1.82

Cohen J. (1988). Statistical power analysis for the behavioral sciences (2nd ed.). Hillsdale, NJ: Erlbaum.

Cresswell, S. L., \& Eklund, R. C. (2006). The convergent and discriminant validity of burnout measures in sport: A multitrait multi-method analysis. Journal of Sports Sciences, 24, 209-220. doi:10.1080/02640410500131431

Csillik, A. (2015). Positive motivational interviewing: Activating clients' strengths and intrinsic motivation to change. Journal of Contemporary Psychotherapy, 45, 119-128. doi:10.1007/s10879-014-9288-6

Diener, E. D., Emmons, R. A., Larsen, R. J., \& Griffin, S. (1985). The Satisfaction with Life Scale. Journal of Personality Assessment, 49, 71-75. doi:10.1207/s15327752jpa4901_13

Diener, E., Wirtz, D., Tov, W., Kim-Prieto, C., Choi, D.-W., Oishi, S., \& Biswas-Diener, R. (2010). New well-being measures: Short scales to assess flourishing and positive and negative feelings. Social Indicators Research, 97, 143-156. doi:10.1007/s11205-009-9493-y

Feldman, G., Hayes, A., Kumar, S., Greeson, J., \& Laurenceau, J.P. (2007). Mindfulness and emotion regulation: The development and initial validation of the Cognitive and Affective Mindfulness Scale-Revised (CAMSR). Journal of Psychopathology \& Behavioral Assessment, 29, 177-190. doi: 10.1007/s10862-006-9035-8

Fledderus, M., Oude Voshaar, M. A., Ten Klooster, P. M., \& Bohlmeijer, E. T. (2012). Further evaluation of the psychometric properties of the Acceptance and Action Questionnaire-II. Psychological Assessment, 24, 925936. doi:10.1037/a0028200

Gardner, F. L., \& Moore, Z. E. (2004). A mindfulness-acceptance-commitment based approach to performance enhancement: Theoretical considerations. Behavior Therapy, 35, 707-723. doi:10.1016/S0005-7894(04)80016-9

Gardner, F. L., \& Moore, Z. E. (2007). The psychology of enhancing human performance: The mindfulnessacceptance-commitment (MAC) approach. New York, NY: Springer.

Gardner, F. L., \& Moore, Z. E. (2012). Mindfulness and acceptance models in sport psychology: A decade of basic and applied scientific advancements. Canadian Psychology, 53, 309-318. doi:10.1037/a0030220

Goodman, F. R., Kashdan, T. B., Mallard, T. T., \& Schumann, M. (2014). A brief mindfulness and yoga intervention with an entire NCAA Division I athletic team: An initial investigation. Psychology of Consciousness: Theory, Research, and Practice, 1, 339-356. doi:10.1037/cns0000022

Grossman, P., Niemann, L., Schmidt, S., \& Walach, H. (2004). Mindfulness-based stress reduction and health benefits: A meta-analysis. Journal of Psychosomatic Research, 57, 35-43. doi:10.1016/S0022-3999(03)00573-7

Hayes, S. C., Strosahl, K., \& Wilson, K. G. (1999). Acceptance and commitment therapy: An experiential approach to behavior change. New York, NY: The Guilford Press.

Hettema, J., Steele, J., \& Miller, W. R. (2005). Motivational interviewing. Annual Review of Clinical Psychology, 1, 91-111. doi:10.1146/annurev.clinpsy.1.102803.143833

Ho, M. S., Appleton, P. R., Cumming, J., \& Duda, J. L. (2015). Examining the relationship between perfectionism dimensions and burning out symptoms in deaf and hearing athletes. Journal of Clinical Sport Psychology, 9, 156-172. doi:10.1123/jcsp.2014-0035

International Paralympic Committee. (2016). International Standard for Eligible Impairments. Retrieved from

https://www.paralympic.org/sites/default/files/document/161004145727129_2016_10_04_International_Standar d for Eligible Impairments 1.pdf

Johnston, M., Foster, M., Shennan, J., Starkey, N. J., \& Johnson, A. (2010). The effectiveness of an acceptance and commitment therapy self-help intervention for chronic pain. The Clinical Journal of Pain, 26, 393-402. doi:10.1097/AJP.0b013e3181cf59ce

Kendall, M. G., \& Stuart, A. (1958). The advanced theory of statistics. NY: Hafner.

Keyes, C. L. M. (1998). Social well-being. Social Psychology Quarterly, 61, 121-140. doi:10.2307/2787065

Keyes, C. L., \& Shapiro, A. D. (2004). Social well-being in the United States: A descriptive epidemiology. In O. G. Brim, C. D. Ryff, \& R. C. Kessler (Eds.), How healthy are we? A national study of well-being at midlife (pp. 350-372). Chicago, IL: University of Chicago Press. 
Lemyre, P. N., Hall, H. K., \& Roberts, G. C. (2008). A social cognitive approach to burnout in elite athletes. Scandinavian Journal of Medicine \& Science in Sports, 18, 221-234. doi:10.1111/j.1600-0838.2007.00671.x

Lundqvist, C. (2011). Well-being in competitive sports-The feel-good factor? A review of conceptual considerations of well-being. International Review of Sport and Exercise Psychology, 4, 109-127. https://doi.org/10.1080/1750984X.2011.584067.

Lundqvist, C., \& Sandin, F. (2014). Well-Being in elite sport: Dimensions of hedonic and eudaimonic well-being among elite orienteers. Sport Psychologist, 28,, 245-254, doi:10.1123/tsp.2013-0024

Lundqvist, C., \& Raglin, J. (2015). The relationship of basic need satisfaction, motivational climate and personality to well-being and stress patterns among elite athletes: An explorative study. Motivation and Emotion, 39, 237-246. doi: 10.1007/s11031-014-9444-z

Macdougall, H., O'Halloran, P., Shields, N., \& Sherry, E. (2015). Comparing the well-being of para and Olympic sport athletes: a systematic review. Adapted Physical Activity Quarterly, 32(3), 256-276.

Macdougall, H., O’Halloran, P., Sherry, E., \& Shields, N. (2016). Needs and strengths of Australian para-athletes: identifying their subjective psychological, social, and physical health and well-being. The Sport Psychologist, 30(1), 1-12.

Martin, J. J. (2012). Mental preparation for the 2014 Winter Paralympic Games. Clinical Journal of Sport Medicine, 22, 70-73. doi: 10.1097/JSM.0b013e31824204cc

Mason, V. L., Mathias, B., \& Skevington, S. M. (2008). Accepting low back pain: Is it related to a good quality of life? The Clinical Journal of Pain, 24, 22-29. doi:10.1097/AJP.0b013e318156d94f

Miller, W. R., \& Rollnick, S. (2012). Motivational interviewing: Preparing people for change (3rd ed.). New York, NY: The Guilford Press.

Moyers, T. B., Manuel, J. K., \& Ernst, D. (2015). Motivational interviewing treatment integrity coding manual 4.2.1. Retrieved from http://casaa.unm.edu/download/MITI4 2.pdf

Naar-King S, Earnshaw P, Breckon J. (2013). Toward a universal maintenance intervention: Integrating cognitive behavioral treatment with motivational interviewing for maintenance of behavior change. Journal of Cognitive Psychotherapy, 27, 126-137. doi: 10.1891/0889-8391.27.2.126

Osman, A., Barrios, F. X., Gutierrez, P. M., Kopper, B. A., Merrifield, T., \& Grittmann, L. (2000). The pain catastrophizing scale: Further psychometric evaluation with adult samples. Journal of Behavioral Medicine, 23, 351-365. doi:10.1023/A:1005548801037

Pavot, W., \& Diener, E. (1993). Review of the Satisfaction with Life Scale. Psychological Assessment, 5, $164-172$. doi:10.1037/1040-3590.5.2.164

Powell, A. J., \& Myers, T. D. (2017). Developing mental toughness: lessons from paralympians. Frontiers in Psychology, 8, 1270. doi: 10.3389/fpsyg.2017.01270

Raedeke, T.D., \& Smith, A.L. (2001). Development and preliminary validation of an athlete burnout measure. Journal of Sport \& Exercise Psychology, 23, 281-306.

Reinboth, M., \& Duda, J. L. (2004). The motivational climate, perceived ability, and athletes' psychological and physical well-being. The Sport Psychologist, 18(3), 237-251.

Ryan, R. M., \& Deci, E. L. (2001). On happiness and human potentials: A review of research on hedonic and eudaimonic well-being. Annual review of psychology, 52(1), 141-166.

Ryff, C. D. (1989). Happiness is everything, or is it? Explorations on the meaning of psychological well-being. Journal of Personality and Social Psychology, 57, 1069-1081. doi:10.1037/0022-3514.57.6.1069

Ryff, C., Almeida, D. M., Ayanian, J. S., Carr, D. S., Cleary, P. D., Coe, C.,.... Williams, D. (2012). National Survey of Midlife Development in the United States (MIDUS II), 2004-2006. Ann Arbor, MI: Inter-university Consortium for Political \& Social Research.

Segal, Z. V., Williams, J. M. G., \& Teasdale, J. D. (2002). Mindfulness-based cognitive therapy for depression. New York, NY: The Guilford Press.

Sullivan, M. J., Bishop, S. R., \& Pivik, J. (1995). The pain catastrophizing scale: Development and validation. Psychological Assessment, 7, 524-532. doi:10.1037/1040-3590.7.4.524

Thompson, R. W., Kaufman, K. A., De Petrillo, L. A., Glass, C. R., \& Arnkoff, D. B. (2011). One year follow-up of mindful sport performance enhancement (MSPE) with archers, golfers, and runners. Journal of Clinical Sport Psychology, 5, 99-116. doi:10.1123/jcsp.5.2.99

Veehof, M.M., Oskam, M., Schreurs, K.M.G., Bohlmeijer, E.T. (2011). Acceptance-based interventions for the treatment of chronic pain: A systematic review and meta-analysis. $P A I N \circledast$, 152, 533-542. doi.org/10.1016/j.pain.2010.11.002. 


\section{Appendix A}

\section{Assessment Interview}

The assessment interview was developed by Gardner and Moore (2004) and included the Multilevel Classification System for Sport Psychology (MCS-SP). The MCS-SP is a systematic, holistic, and comprehensive assessment that culminates in an individualised intervention plan, and includes an initial case formulation with 10 elements: (a) contextual performance demands, (b) skill level, (c) situational demands, (d) transitional and developmental issues, (e) psychological characteristics/performance and non-performance schemas, (f) attentional focus, (g) cognitive responses, (h) affective responses, (i) behavioural responses, and (j) readiness for change and level of reactance. To assist with the initial case formulation, athletes completed the Performance Classification Questionnaire (Wolanin, 2005), which included a 10-item measure of sport-related behaviours using a 5-point scale (e.g., "My thoughts or feelings make it difficult for me to perform well"). The Performance Classification Questionnaire is specifically used to identify the presence or absence of performance dysfunction. The cut-off score of 30 indicated that athletes met criteria for performance dysfunction, as opposed to performance development. Performance dysfunction refers to athletes whose performance has been delayed or impaired due to subclinical psychological symptoms or other difficulties. From the initial case formulation, MCS-SP classification, an extensive case description was compiled and an appropriate MAC intervention program developed. During the first session, athletes received the MAC program book, complete with all the handouts, activities, psychoeducation, and resources for the program. Athletes were asked to bring this book to each session to record their answers, reflections, and any other notes.

\section{Session 2: Preparing the Athlete With Psychoeducation}

The session began (and each session thereafter) with a mindfulness metaphor (e.g., the sky and the weather) to help athletes understand the concept. A layman's introduction of the theoretical rationale for the program was then presented along with an explanation of how performance can be enhanced through skills such as awareness (mindfulness), acceptance, commitment, and poise. Emphasis was placed on not controlling internal experiences and connecting this with a time when the athlete performed well yet had unhelpful thoughts, feelings, or emotions. The session concluded with an introduction of the brief centring exercise whereby athlete participants attended to their breath, switched their attention to their surroundings, and then back to their body. The purpose of this exercise was to learn how to switch attention between internal and external experiences. At the conclusion of the session (and each session of the program), the athlete was asked to complete the "What I Have Learned" exercise as soon as possible after the session. This exercise was designed to promote reflection, and thereby self-awareness. At the end of the session, athletes were asked to read the "Preparing for MAC" document, which contained an overview of the basic essentials for beginning the program, as well as practice the brief centring exercise. Athletes were sent audio files for each mindful activity to encourage their practice.

\section{Session 3: Introducing Mindfulness and Cognitive Defusion}

The format for the beginning of each session was the completion of a mindfulness activity, a discussion of the "What I Have Learned" form, a discussion of any between-session activities, and a check for any questions/uncertainties regarding the previous session; the mindful activity for Session 3 was mindful eating. The fundamental concepts of mindfulness, cognitive fusion, and defusion were then explored. Mindfulness was explained through both Eastern and Western definitions (e.g., paying attention with curiosity, flexibility, and openness); how mindfulness begins the process of cognitive defusion, which assists in task-focused attention, was also discussed. Cognitive fusion was described as responding to thoughts as absolute truths that must be responded to in some way, whereas cognitive defusion uses mindfulness skills to unhook from internal experiences thereby allowing control over behaviour. Each session concluded with another mindful activity, such as the 
brief centring exercise. Before the next session, athletes were asked to complete the "Washing the Dish Mindfully" activity.

\section{Session 4: Introducing Values and Values-Driven Behaviour}

Through metaphor, emotion-driven behaviour and value-driven behaviour were explored. Goals and values were also differentiated, where goals were described as the destination and values as the journey. Athletes identified their performance and life values, and were asked to complete the "Performance Values" exercise before the next session to enable reflection on how their values were relevant to different domains of their performance. The function of emotions was also explored; emphasis was placed on how cognitive fusion - not emotion - is typically a barrier to performance. The session concluded with the "Mindfulness of the Breath" exercise, where athletes continuously focused on their breath while noticing the flow of air going in and out of the body and the abdomen rising and falling. Athletes were asked to apply mindfulness to a simple training activity, and complete the "Given Up for Emotions" exercise; this exercise was intended to allow the athlete to explore the personal and performance-related consequences of expending unnecessary effort to control and/or eliminate emotions, as opposed to focusing on values-directed choices and actions.

\section{Session 5: Introducing Acceptance}

The concepts introduced in this session were experiential avoidance and acceptance. Experiential avoidance was explained as behaviour that seeks to avoid or get rid of unhelpful thoughts or feelings, and inhibits value-driven behaviour. Athletes completed the "Join the DOTS" activity in which experiential avoidance techniques (i.e., distraction, opting out, thinking strategies, substances/other strategies) were identified. Athletes then classified these strategies as harmful or not harmful. The "Pushing the Paper Away" exercise was then used to explain experiential acceptance-the experience of negative internal states without judgment (Hayes et al., 1999) - and to describe how experiential acceptance mitigates experiential avoidance. Before the next session, athletes were asked to complete the "Emotion and Performance Interference" exercise in which emotions interfering with performance were assessed. The session concluded with a mindful activity and athletes were asked to practice the "Leaves on a Stream" mindfulness activity.

\section{Session 6: Enhancing Commitment}

Similar to Goodman et al. (2014), the concepts of fixed and growth mindsets were introduced to initiate a discussion about commitment. Fixed mindsets were explained as an orientation toward avoiding challenges, easily giving up, and ignoring feedback, whereas growth mindsets were explained as an orientation toward embracing challenges, persisting through setbacks, and learning from experiences. Athletes were asked to provide examples from their own performance of fixed and/or growth mindsets. Athletes were also taken through the resiliency formula and a reframe exercise to help build commitment and acceptance. Before the next session, athletes were asked to complete the "Committing to Performance Values" exercise in which a performance value-and short-term and long-term goals associated with that value-and behavioural changes required to achieve desired performance were identified. If possible, athletes were asked to extend mindfulness into competition or build upon previous mindful activities within their training. The session concluded with a brief body scan, in which athletes attended to their breath then progressively moved their attention from one area of the body to another; athletes were sent brief and long body scan audio files.

\section{Session 7: Skill Consolidation and Poise - Combining Mindfulness, Acceptance, and Commitment}

Athletes completed the "Task-Focused Attention" exercise in which they learned how to redirect their attention from internal processes (i.e., emotions or thoughts) to the external task of listening to a 2- to 3-minute story from the instructor. Athletes were required to recount at least $50 \%$ of the story 
details before the difficulty of the exercise was increased (no eye contact, eye contact, and level of details within the story). The relevancy of this skill to performance was explored, and athletes were asked to practice this skill over the next week. The physiology behind breath, and how breath connects the mind and body, was also briefly explored in order to promote athletes' understanding of the experiential exercises completed throughout the program. Athletes were asked to prepare a creative reflection piece before the final session, and prepare a 2-minute mindfulness practice session in which they were the instructor.

\section{Session 8: Maintaining and Enhancing Mindfulness, Acceptance, and Commitment}

The session began with the athlete presenting her or his creative reflection piece and mindfulness practice in which s/he was the instructor. The key pillars of performance, and the program, were reviewed. Athletes completed similar performance evaluation exercises as found in Session 1 and 2; the results of these evaluations were then compared and discussed. Athletes completed the postMAC practice plan exercise to assess performance values, goals, obstacles, and avoidant behaviours, and to set goals for future practice. They were encouraged to use the plan to monitor progress, identify areas for improvement, and use their social support networks for support. To conclude the program, athletes were presented with a certificate of achievement for completing the program.

(C) 2019 by the authors. Submitted for possible open access publication under the terms and conditions of the Creative Commons Attribution (CC BY) license (http://creativecommons.org/licenses/by/4.0/). 